Pacific Journal of Mathematic 


\title{
CANCELLATION OF MODULES AND GROUPS AND STABLE RANGE OF ENDOMORPHISM RINGS
}

\author{
R. B. WARFIELD, JR.
}

In this paper, various cancellation properties for a module are shown to follow from facts about the stable range of its endomorphism ring, and a number of applications to the structure of modules, Abelian groups, and complex tori are given.

In the first section we introduce a property which we call the $n$-substitution property for a module, which holds if and only if the integer $n$ is in the stable range of its endomorphism ring. Direct sums and summands of modules with this property are studied, and a slight generalization of Vasershtein's theorem on the stable range of matrix rings is obtained. In the second section two strong cancellation properties for a module are shown to be equivalent to one being in the stable range of its endomorphism ring, and an application is obtained concerning modules whose endomorphism ring modulo its radical is von Neumann regular. Bass showed that if $S$ is a commutative $J$-Noetherian ring of $J$-dimension $d$, and $R$ is a finite $S$-algebra, then $d+1$ is in the stable range for $R$. If $S$ is not Noetherian, this is not a strong enough result to apply to endomorphism rings, and it is improved in the third section to say that the endomorphism ring of any finitely presented module over such a ring has $d+1$ in the stable range. In section four are two stronger results, one concerning finitely presented modules over rings of Krull dimension one in the classical sense (in terms of chains of prime ideals) and the other concerning projective modules over rings having Krull dimension one in the noncommutative sense. The final section contains several applications to torsion-free Abelian groups, modules over valuation rings, and complex tori. It is shown that a torsion-free algebra of finite rank over a semilocal principal ideal domain or over a (possibly non-Noetherian) valuation ring has one in the stable range. This implies cancellation theorems for torsion-free Abelian groups of finite rank satisfying certain divisibility conditions and also for torsion-free modules of finite rank over a valuation ring. It is shown that two is in the stable range for any torsion-free algebra of finite rank over a principal ideal domain. As an application, it is shown that two torsion-free Abelian groups $A$ and $B$ of finite rank are of the same genus if and only if for some positive integer $n, A^{n} \cong B^{n}$, so that, in particular, if $A, B$, and $C$ are torsion-free groups of finite rank and $A \oplus B \cong A \oplus C$, then for some positive integer $n, B^{n} \cong C^{n}$. There are corresponding ap- 
plications to complex tori, and, in particular, if $A, B$, and $C$ are tori with $A \times B \cong A \times C$, then for some positive integer $n, B^{n} \cong C^{n}$.

All modules in this paper are right modules, on which homomorphisms and endomorphisms act on the left, though we may occasionally vary this convention for modules over commutative rings. All rings have identity, and by the radical of ring $E$, we will always mean the Jacobson radical, denoted $J(E)$, unless we specify otherwise. The author has profited from a number of conversations with K. R. Goodearl about the material in this paper. This paper was completed while the author was visiting at the University of Leeds, and the author is grateful to the Mathematics faculty at that institution for their hospitality. The research was supported in part by a grant from the National Science Foundation.

\section{Stable range and the $n$-substitution property.}

Definition 1.1. A module $A$ has the $n$-substitution property if for every split epimorphism $M=\left(\bigoplus_{i=1}^{n} A\right) \oplus H \rightarrow A$ there is a splitting $\phi: A \rightarrow M$ such that $M=\phi(A) \oplus L \oplus H$ with $L \subseteq \bigoplus_{i=1}^{n} A$.

THEOREM 1.2. If $A$ has the n-substitution property and $A \oplus X \cong A \oplus Y$, and $X$ has a summand isomorphic to $\bigoplus_{i=1}^{n} A$, then $X \cong Y$.

Proof. Let $X=B_{1} \oplus \cdots \oplus B_{n} \oplus X^{\prime}$, where $B_{i} \cong A(1 \leqq i \leqq n)$, and let $B=\bigoplus_{i=1}^{n} B_{i}$. We have a split epimorphism $B \oplus A \oplus X^{\prime} \rightarrow A$ with kernel $Y^{\prime}, Y^{\prime} \cong Y$. By hypothesis, there is a splitting $\phi: A \rightarrow$ $B \oplus A \oplus X^{\prime}$ such that $B \oplus A \oplus X^{\prime}=\phi(A) \oplus L \oplus A \oplus X^{\prime}$ where $L \cong B$. Since $\phi(A) \oplus L \cong B$, and $A \cong \phi(A)$, it follows that $L \oplus A \oplus$ $X^{\prime} \cong X$. Since we also have $B \oplus A \oplus X^{\prime}=\phi(A) \oplus Y^{\prime}$, and $Y^{\prime} \cong Y$, it follows that $Y \cong X$.

THEOREM 1.3. Let $A$ be a module with the n-substitution property and $E$ its endomorphism ring. If $A \oplus X \cong A \oplus Y$ and $X \cong\left(\bigoplus_{i=1}^{n-1} A\right) \oplus X^{\prime}$, then $Y \cong X^{\prime} \oplus L$, where $L \oplus A \cong \bigoplus_{i=1}^{n} A$. We can conclude from this that $L \cong \bigoplus_{i=1}^{n-1} A$ (so that $X \cong Y$ ) if and only if for any finitely generated projective $E$-module $P$ such that $P \oplus E=\bigoplus_{i=1}^{n} E$, we have $P \cong \bigoplus_{i=1}^{n-1} E$.

Proof. The first statement is proved just as the statement of the previous theorem. There is an obvious equivalence of categories between finitely generated projective $E$-modules and summands of finite direct sums of copies of $A$ (given by $M_{R} \rightarrow \operatorname{Hom}(A, M)_{E}$ and $\left.P_{E} \rightarrow P_{E} \otimes_{E} A_{R}\right)$ which establishes the second statement. 
REMARK. To see what this means, suppose that $A$ is a module with $\operatorname{End}(A) \cong Z$. Anticipating results, we simply state that this implies that $A$ has the 2-substitution property. Since projective $Z$-modules are free, we conclude from 1.3 that $A \oplus A \oplus X \cong A \oplus Y$ implies $Y \cong A \oplus X$.

Definition 1.4. A sequence $\left(a_{1}, \cdots, a_{r}\right)$ of elements of a ring $E$ is a right unmodular row if there are elements $x_{i} \in E$ with $\sum a_{i} x_{i}=1$. Similarly, $\left(a_{1}, \cdots, a_{r}\right)$ is a left unimodular row if there are elements $x_{i} \in E$ with $\sum x_{i} a_{i}=1$.

Definition 1.5. An integer $n>0$ is said to be in the stable range for a ring $E$ if for every right unimodular row $\left(a_{1}, \cdots, a_{r}\right)$, $(r>n)$ of elements of $E$, there are elements $b_{1}, \cdots, b_{r-1}$ in $E$ such that $\left(a_{1}+a_{r} b_{1}, \cdots, a_{r-1}+a_{r} b_{r-1}\right)$ is right unimodular.

THEOREM 1.6. The following properties are equivalent for a ring $E$ with identity, and an integer $n, n>0$.

1. For every right unimodular row $\left(a_{1}, \cdots, a_{r}\right),(r>n)$ of elements of $E$, there are elements $b_{1}, \cdots, b_{r-1}$ in $E$ such that $\left(a_{1}+a_{r} b_{1}, \cdots, a_{r-1}+a_{r} b_{r-1}\right)$ is right unimodular.

$1^{\prime}$. For every left unimodular row $\left(a_{1}, \cdots, a_{r}\right), r>n$, of elements of $E$, there are elements $b_{1}, \cdots, b_{r-1}$ of $E$ such that $(a+$ $\left.b_{1} a_{r}, \cdots, a_{r-1}+b_{r-1} a_{r}\right)$ is left unimodular.

2. For every right unimodular row $\left(a_{1}, \cdots, a_{r}\right), r>n$, there are elements $x_{i}(1 \leqq i \leqq r)$ such that $\sum a_{i} x_{i}=1$ and $\left(x_{1}, \cdots, x_{r-1}\right)$ is left unimodular.

2'. For every left unimodular row $\left(a_{1}, \cdots, a_{r}\right), r>n$, there are elements $x_{i}(1 \leqq i \leqq r)$ such that $\sum x_{i} a_{i}=1$ and $\left(x_{1}, \cdots, x_{r-1}\right)$ is right unimodular.

3. If $A$ is a right module over a ring $R$ and $E$ is the endomorphism ring of $A$ (acting on the left) then $A$ has the n-substitution property.

$3^{\prime}$. If $A$ is a right module over a ring $R$ and $E$ is the endomorphism ring of $A$ (acting on the left), and if $M$ is a right module with decompositions

$$
M=A_{1} \oplus \cdots \oplus A_{n} \oplus H=A_{0} \oplus D
$$

with $A \cong A_{i},(0 \leqq i \leqq n)$ then there are submodules $K \cong M$ and $L \subseteq A_{1} \oplus \cdots \oplus A_{n}$ such that $M=A_{0} \oplus K=L \oplus K$.

REMARK. Condition $\left(3^{\prime}\right)$ is dual to condition (3) and can be obtained from (3) by changing the split epimorphism to a split monomorphism and making other changes accordingly. We chose not to 
emphasize the duality because the present statement seems clearer. Throughout this theorem we have restricted ourselves to right modules with endomorphisms acting on the left, but because of the symmetry of the results, one does not have to be careful about how the endomorphisms act.

We isolate part of the proof of the theorem for later reference.

LEMMA 1.7. If $E$ is a ring and $\left(a_{1}, \cdots, a_{r}\right)$ a right unimodular row in $E$, then conditions (1) and (2) of Theorem 1.6 for that particular row are equivalent.

Proof. If $\left(a_{1}+a_{r} b_{1}, \cdots, a_{r-1}+a_{r} b_{r-1}\right)$ is right unimodular, and $\sum\left(a_{i}+a_{r} b_{i}\right) x_{i}=1$, then $\left(x_{1}, \cdots, x_{r-1}\right)$ is left unimodular, and $a_{1} x_{1}+$ $\cdots+a_{r-1} x_{r-1}+a_{r}\left(\sum b_{i} x_{i}\right)=1$, so (2) holds. Conversely, if $\sum a_{i} x_{i}=1$ and $\left(x_{1}, \cdots, x_{r-1}\right)$ is left unimodular, and $c_{1} x_{1}+\cdots+c_{r-1} x_{r-1}=1$, then if we let $b_{i}=x_{r} c_{i}$ then we easily calculate that $\left(a_{1}+a_{r} b_{1}\right) x_{1}+$ $\cdots+\left(a_{r-1}+a_{r} b_{r-1}\right) x_{r-1}=1$.

Proof of theorem. Lemma 1.7 shows that (1) and (2) are equivalent as are (1') and $\left(2^{\prime}\right)$. We next show that (2) implies (3). If $\pi=\left(\pi_{1}, \cdots, \pi_{n}, f\right): A \oplus \cdots \oplus A \oplus H \rightarrow A$ is a split epimorphism, and $\phi=\left(\phi_{1}, \cdots, \phi_{n}, g\right): A \rightarrow A \oplus \cdots \oplus A \oplus H$ is a splitting, then $\pi \phi=1$, so $\pi_{1} \phi_{1}+\cdots+\pi_{n} \phi_{n}+f g=1$. We apply (2) to the right unimodular row $\left(\pi_{1}, \cdots, \pi_{n}, f g\right)$ in $E$, obtaining elements $\Psi_{i}(1 \leqq i \leqq n+1)$ such that $\left(\Psi_{1}, \cdots, \Psi_{n}\right)$ is left unimodular and $\pi_{1} \Psi_{1}+\cdots+\pi_{n} \Psi_{n}+(f g) \Psi_{n+1}=1$. We let $\Psi: A \rightarrow\left(\bigoplus_{i=1}^{n} A\right) \oplus H$ be defind by $\Psi=\left(\Psi_{1}, \cdots, \Psi_{n}, g \Psi_{n+1}\right)$. $\Psi$ is clearly a right inverse for $\pi$. Since $\left(\Psi_{1}, \cdots, \Psi_{n}\right)$ is left unimodular, there are elements $\theta_{i}$ of $E$ such that $\theta_{1} \Psi_{1}+\cdots+\theta_{n} \Psi_{n}=1$. We now find another left inverse for $\Psi$, namely the map $\theta=\left(\theta_{1}, \cdots, \theta_{n}, 0\right)$ : $\left(\bigoplus_{i=1}^{n} A\right) \oplus H \rightarrow A$. Clearly, $\operatorname{Ker}(\theta)=L \oplus H$ where $L \subseteq\left(\bigoplus_{i=1}^{n} A\right)$, and we have proved that $A$ has the $n$-substitution property.

We next show that (3) implies (2). We regard the right unimodular row $\left(a_{1}, \cdots, a_{r}\right)$ of elements of $E$ as a split epimorphism $\left(\bigoplus_{i=1}^{r} A\right) \rightarrow A$. A splitting is given by a row $\left(x_{1}, \cdots, x_{r}\right)$ of elements of $E$ such that $\sum a_{i} x_{i}=1$. Condition (3) says that we can find such a row, such that if $\phi$ is the corresponding homomorphism $A \rightarrow$ $\left(\bigoplus_{i=1}^{r} A\right)$, then there is a submodule $L \cong\left(\bigoplus_{i=1}^{n} A\right)$ with $\bigoplus_{i=1}^{r} A=$ $\phi(A) \oplus L \oplus\left(\bigoplus_{i=n+1}^{r} A\right)$. If $\phi$ has this property, then $\phi$ has a left inverse $\beta$ such that $\operatorname{Ker}(\beta)=L \oplus\left(\bigoplus_{i=n+1}^{r} A\right)$. Such a $\beta$ : $\bigoplus_{i=1}^{r} A \rightarrow A$ is given by a row of the form $\left(b_{1}, \cdots, b_{n}, 0, \cdots, 0\right)$. Hence $\left(x_{1}, \cdots, x_{n}\right)$ is left unimodular, which proves (2).

The equivalence of $\left(1^{\prime}\right),\left(2^{\prime}\right)$ and $\left(3^{\prime}\right)$ is directly parallel to that of (1), (2) and (3) (in fact, they are the same statements in the opposite ring), so the theorem will be proved if we can show the 
equivalence of (2) and $\left(2^{\prime}\right)$. The proof that (2) and (3) are equivalent makes it clear that $A$ has the $n$-substitution property if and only if $A$ has this property in the special case in which $H \cong A$. Similarly, in establishing property $\left(3^{\prime}\right)$, we may assume $H \cong A$. We therefore let $M=A_{1} \oplus \cdots \oplus A_{n+1}=A_{0} \oplus D$, with $A_{i} \cong A,(0 \leqq i \leqq$ $n+1)$, we assume $A$ has the $n$-substiution property, and we prove that condition $\left(3^{\prime}\right)$ holds, that is, there are submodules $L$ and $K$ of $M$ such that $L \subseteq A_{1} \oplus \cdots \oplus A_{n}$ and $A_{0} \oplus K=M=L \oplus K$. By Theorem 1.2, we know that $D \cong\left(\bigoplus_{i=1}^{n} A\right)$. We therefore can apply the $n$-substitution property for $A$ to the split epimorphism $A_{0} \oplus D \rightarrow$ $A_{n+1}$ (with kernel $A_{1} \oplus \cdots \oplus A_{n}$ ) to obtain a splitting $\phi: A_{n+1} \rightarrow M$ with $\phi\left(A_{n+1}\right)=F$, such that $M=A_{1} \oplus \cdots \oplus A_{n} \oplus F=A_{0} \oplus D^{\prime} \oplus F$ where $D^{\prime} \cong D$. We let $K=D^{\prime} \oplus F$. If $L=\left(A_{0} \oplus F\right) \cap\left(A_{1} \oplus \cdots \oplus\right.$ $\left.A_{n}\right)$, it is routine that $M=L \oplus K$. This verifies that condition $\left(3^{\prime}\right)$ holds for $A$. In particular this shows that (2) implies $\left(2^{\prime}\right)$, and passing to opposite rings, this implies that $\left(2^{\prime}\right)$ implies (2), so we have completed the proof of 1.6 .

CoROLlary 1.8. If $M$ is a module with endomorphism ring $E$, then the following properties are equivalent:

(1) $M$ has the $n$-substitution property.

(2) $E$ has the n-substitution property (as an E-module).

(3) $E$ has the n-substitution property in the category of finitely generated projective E-modules.

This is clear from the above theorem and its proof. The $n$-substitution property therefore resembles the finite exchange property, for which the equivalence of (1) and (2) is proved in [37], and the equivalence of (1) and (3) follows from the proof of the main theorem in [25].

Theorem 1.9. If $A$ is a module and $A=B \oplus C$, then $A$ has the $n$-substitution property if $B$ and $C$ do.

Proof. Let $M=A_{1} \oplus \cdots \oplus A_{n} \oplus H=A_{0} \oplus K$, where $A_{i} \cong A$, $0 \leqq i \leqq n$. We must find submodules $A^{\prime}$ and $L$ of $M$ such that $M=A^{\prime} \oplus K=A^{\prime} \oplus L \oplus H$, where $L \cong A_{1} \oplus \cdots A_{n}$. Letting $A_{i}=$ $B_{i} \oplus C_{i}$, with $B_{i} \cong B, C_{i} \cong C,(0 \leqq i \leqq n)$, and applying the $n$-substitution property for $B$, we obtain submodules $B^{\prime} \subseteq M$ and $L^{\prime} \subseteq B_{1} \oplus$ $\cdots \oplus B_{n}$ such that

$$
M=B^{\prime} \oplus C_{0} \oplus K=B^{\prime} \oplus L^{\prime} \oplus C_{1} \oplus \cdots C_{n} \oplus H .
$$

Using these decompositions, and applying the $n$-substitution property for $C$, we obtain submodules, $C^{\prime} \subseteq M$ and $L^{\prime \prime} \subseteq C_{1} \oplus \cdots \oplus C_{n}$ such 
that

$$
M=B^{\prime} \oplus C^{\prime} \oplus K=B^{\prime} \oplus C^{\prime} \oplus L^{\prime} \oplus L^{\prime \prime} \oplus H .
$$

Letting $A^{\prime}=B^{\prime} \oplus C^{\prime}$ and $L=L^{\prime} \oplus L^{\prime \prime}$, we obtain the desired result.

CoROLlary 1.10. If $n$ is in the stable range for a ring $E$, then it is in the stable range for a matrix ring over $E$.

Remark. If a module $M$ has the 1-substitution property, then so does any summand of $M$, as is shown in Fuchs [9]. It follows that the property of having 1 is the stable range is a Morita invariant of rings. This is not the case with the higher stable range conditions, as was first pointed out by Vasershtein in [34]. Vasershtein gives a formula for the stable range of a matrix ring which implies that if $R$ is a ring and $n$ is in the stable range for $R$, then 2 is in the stable range for $M_{n-1}(R)$. The module theoretic approach gives a smoother treatment of this along with a slight generalization.

THEOREM 1.11. Let $A$ be a module with the n-substitution property and suppose that $n$ is the smallest integer for which this is true. If $k>0$ and $m \geqq n-1$, and

$$
M=A_{1} \oplus \cdots \oplus A_{k} \oplus K=A_{1}^{\prime} \oplus \cdots \oplus A_{k+m}^{\prime} \oplus L
$$

where $A_{i} \cong A \cong A_{j}^{\prime}$ for all $i$ and $j$, then there is a submodule $B \cong M$ such that

$$
M=B \oplus K=B \oplus C \oplus L
$$

with $C \leqq A_{1}^{\prime} \oplus \cdots \oplus A_{k+m}^{\prime}$. Conversely, if $n$ and $k$ are as before and if $m$ is an integer for which the above statement always holds, then $m \geqq n-1$.

Proof. If $m \geqq 1$ the proof of the existence of $B$ is an easy iterative argument from the definition and the argument used to prove 1.2. To prove the sharpness of the estimate, we suppose that $k$ is fixed and that the result is true for some integer $m$, and suppose

$$
N=A_{1} \oplus K=A_{1}^{\prime} \oplus \cdots \oplus A_{m+1}^{\prime} \oplus L
$$

where $A_{1} \cong A \cong A_{i}^{\prime}, 1 \leqq i \leqq m$. We let $M=A^{k-1} \oplus N$, and conclude from the condition that there is a submodule $B$ of $M$ with

$$
M=B \oplus K=B \oplus C \oplus L,
$$


where $C \leqq A^{k-1} \oplus A_{1}^{\prime} \oplus \cdots \oplus A_{m+1}^{\prime}$. It follows that

$$
N=(B \cap N) \oplus K=((B \oplus C) \cap N) \oplus L .
$$

Since $B \cap N \subseteq(B \oplus C) \cap N$, this formula implies that $(B \oplus C) \cap N=$ $(B \cap N) \oplus(B \oplus C) \cap K$. If $\pi$ is the projection of $N$ onto $A_{1}^{\prime} \oplus \cdots \oplus$ $A_{m+1}^{\prime}$ along $L$, then $\pi((B \oplus C) \cap K) \oplus L=((B \oplus C) \cap K) \oplus L$, so

$$
N=(B \cap N) \oplus \pi((B \oplus C) \cap K) \oplus L .
$$

Since this holds for any such $N$, we conclude that $A$ has the $m+1$ substitution property, so $m \geqq n-1$, as desired.

To convert this theorem into a statement on the substitution property for $A^{k}$, or, equivalently, on the stable range of $M_{k}(\operatorname{End}(A)$ ), it is necessary to increase $m$ so that it is a multiple of $k$. Doing this, we obtain the unlikely looking formula first obtained by Vasershtein by a different argument:

CoRollary 1.12. If $n$ is the smallest integer in the stable range for $R$, then the smallest integer in the stable range for $M_{k}(R)$ is $1-[-(n-1) / k]$, where $[r]$ is the greatest integer less than or equal to $r$.

\section{Cancellation and the substitution property.}

THEOREM 2.1. For a module $A$ with endomorphism ring $E$, the following properties are equivalent:

(i) A has the 1-substitution property.

(ii) $E$ has one in the stable range.

(iii) For every module $M$ with decompositions $M=A_{1} \oplus H=$ $A_{2} \oplus K$ with $A \cong A_{1} \cong A_{2}$, there is a submodule $C \cong M$ with

$$
M=A_{1} \oplus C=A_{2} \oplus C .
$$

(iv) For every module $M$ with decompositions $M=A_{1} \oplus H=A_{2} \oplus K$ with $A \cong A_{1} \cong A_{2}$, there is a submodule $C \cong M$ with

$$
M=C \oplus H=C \oplus K
$$

Further, each of these properties implies that every one-sided unit in $E$ is a unit and that every module with endomorphism ring isomorphic to $E$ has the cancellation property.

REMARK. The properties described in (iii) and (iv) are sometimes called (respectively) the common complement and substitution properties. The substitution property was introduced by Fuchs in 
[9]. The substitution property had previously been established by Crawley [5] for countable $p$-groups with finite Ulm invariants. Fuchs showed in [9] that a quasi-projective module $A$ has the substitution property if and only if its endomorphism ring does as a module over itself, a result which 2.1 and 1.6 show holds for all modules. Theorem 2.1 can be thought of as an elaboration of Evans's theorem [8] that a module has the cancellation property if its endomorphism ring has one in the stable range.

Proof. Theorem 1.6 shows that (i) and (ii) are equivalent. We assume (ii) and let the projection onto $A_{2}$ be the split epimorphism, and we obtain a splitting $\phi$ such that $M=\phi(A) \oplus Q \oplus H$ with $\phi(A) \cong A$ and $\phi(A) \oplus Q \cong A$. To show $Q=0$, it suffices to show that $A$ cannot be isomorphic to a proper summand of itself. This is clear if we take the isomorphism $\phi(A) \oplus Q \rightarrow A$ as our spilt epimorphism and apply the 1-substitution property again. This shows that (i) implies (iv), and the converse is trivial. The fact that $A$ has no proper summands isomorphic to itself is equivalent to the statement that one-sided units in $E$ are units, which (together with 1.6) shows that (ii) implies (iii), the converse again being trivial from 1.6. This completes the proof of 2.1.

One may define a cancellation ring to be a ring $E$ such that every module with endomorphism ring isomorphic to $E$ has the cancellation property. No examples of cancellation rings are known which do not have 1 in the stable range. There are, however, modules with the cancellation property whose endomorphism rings do not have 1 in the stable range-the best example being $Z$ - the infinite cyclic group, [4, 35]. However, if $A$ is a noncyclic subgroup of the additive group of rationals such that $\operatorname{End}(A) \cong Z$ (there are many such) then $A$ does not have the cancellation property (see 5.5 below), so $Z$ is not a cancellation ring.

The next result is a well known result due to Bass ([3, Lemma 6.4]) which we include for reference, and because an amusing proof is now available.

LEMMA 2.2. (Bass [3]). If $E$ is a ring such that $E / J(E)$ is Artinian then $E$ has one in the stable range.

Proof. The property clearly holds for $E$ if and only if it holds for $E / J(E)$, so we may assume that $E$ is Artinian and semi-simple. $E$ is then a product of simple rings, and the behavior of the formulae involved shows that we may assume that $E$ is simple. $E$ is then a matrix ring over a division ring. Lemma 1.9 shows that it will suffice to establish the result when $E$ is a division ring, 
which is trivial.

The next lemma is an easy application of 2.2 , and is a special case of [13, Prop. 12(a) and Thm. 18]. However, its proof is much easier than that in [13], and it is needed frequently below, so we include a proof.

LEMma 2.3. Let $S$ be a commutative ring with only a finite number of maximal ideals and let $R$ be an S-algebra which is finitely generated as an $S$-module. Then if $A$ is a finitely generated $R$-module, with endomorphism ring $E$, then $E / J(E)$ is Artinian, so one is in the stable range for $E$ and $A$ has the substitution and common complement properties.

Proof. Let $K$ be the kernel of the natural map $E \rightarrow \operatorname{End}_{S}(A /$ $A J(S)) . \quad E / K$ is an $S / J(S)$-subalgebra of $\operatorname{End}_{S}(A / A J(S))$. Since $S / J(S)$ is a finite product of fields, and $A / A J(S)$ is a finitely generated $S / J(S)$-module, we see that $E / K$ is Artinian, so all we need to show is that $K \cong J(E)$. If $f \in K$, the $1-f$ is surjective modulo $J(S)$, and hence is surjective by Nakayama's lemma. Since $A$ is finitely generated over the commutative ring $S$, it follows from Vasconcelos' lemma [33] that $1-f$ is monic. This shows that $1-f$ is an automorphism for all $f \in K$, so $K \subseteq J(E)$.

This generalizes a result of Evans' in [8] in which $S$ is a Noetherian local ring. In [13], a more general result is obtained, in which it is only required that $S / J(S)$ is von Neumann regular and that for each maximal ideal $M$ of $S, R_{M}$ is finitely generated as an $S_{M}$-module.

$A$ reading of the proof of Bass's lemma given by Swan in [31, 11.8] shows that if $R$ is von Neumann regular and if $R$ has the internal cancellation property $\left(R_{R}=A \oplus B=C \oplus D\right.$ and $A \cong C$ imply $B \cong D$ ) then $R$ has one in the stable range. Conversely, if $R$ has one in the stable range then our previous results and the fact (due to Fuchs [9]) that the substitution property is inherited by summands implies the internal cancellation property. This observation is due to Kaplansky (unpublished) and Fuchs [9]. A ring is unit regular if for every $x \in R$, there is a unit $u$ with $x u x=x$. In [16] is a proof, due to Kaplansky, that a regular ring is unit regular if and only if it has one in the stable range. These remarks summarize the known results on the stable range of von Neumann regular rings, and lead to the next result.

THEOREM 2.4. Let $E$ be a ring with radical $J$ such that idempotents modulo $J$ can be lifted and $E / J$ is von Neumann regular. Then the following properties of $E$ are equivalent: 
(i) $E / J$ is unit regular.

(ii) 1 is in the stable range for $E$.

(iii) every module whose endomorphism ring is isomorphic to $E$ has the cancellation property.

(iv) there exists a ring $R$ and an $R$-module $M$ such that $\operatorname{End}(M) \cong E$ and $M$ has the cancellation property.

REMARK. This shows that at least for these rings, the cancellation property is equivalent to the substitution property.

Before proving this theorem we need some terminology and a lemma. We recall that an additive functor $F: \mathscr{A} \rightarrow \mathscr{B}$ between additive categories is a weak representation equivalence if (i) $F(X) \cong$ $F(Y)$ implies $X \cong Y$; (ii) for each object $X$ of $\mathscr{B}$, there is an object $Y$ of $\mathscr{A}$ with $F(Y) \cong X$. These hypotheses imply $F(X \oplus Y) \cong$ $F(X) \oplus F(Y)$ and that $X$ has the cancellation property in $\mathscr{A}$ if and only if $F(X)$ does in $\mathscr{B}$, and similar statements.

Lemma 2.5. Let $M$ be a module, $E$ its endomorphism ring, $J$ the radical of $E$, and suppose that $E / J$ is von Neumann regular and that idempotents modulo $J$ can be lifted. Then there is a weak representation equivalence from the category of summands of finite direct sums of copies of $M$ to the category of finitely generated projective $E / J$-modules.

Proof. We first note that the functor taking $N_{R}$ to $\operatorname{Hom}(M$, $N)_{E}$ is category equivalence (with inverse taking $P_{E}$ to $P_{E} \otimes_{E} M_{R}$ ) from the category of summands of finite direct sums of copies on $M$ to the category of finitely generated projective $E$-modules. The functor from projective $E$-modules to projective $E / J$ modules takes $P$ to $P / P J$. A standard argument (as in [31, 2.26]) shows that if $P / P J \cong Q / Q J$ then $P \cong Q$ (if $P$ and $Q$ are finitely generated projective $E$-modules), so we need only show that for every finitely generated projective $E / J$-module $Q$ there is a finitely generated projective $E$-module $P$ with $P / P J \cong Q$. Since every projective $E / J$ module is a direct sum of idempotent generated ideals [21], it is enough to verify this when $Q \cong e(E / J)$ for some idempotent $e \in E / J$. By the idempotent lifting hypothesis, we may assume that $e$ is an idempotent of $E$, in which case we can let $P=e E$.

Proof of 2.4. It is clear from our previous remarks that (i) and (ii) are equivalent, and that (ii) implies (iii), which implies (iv). We therefore show (iv) implies (i). By the lemma, if $M$ has the cancellation property, then $E / J$ does in the category of finitely generated projective $E / J$-modules. Let $E / J=A \oplus B=C \oplus D$, with 
$A \cong C$. Adding a copy of $B$ to both sides, we obtain $E / J \oplus B \cong$ $E / J \oplus D$. Hence, if $M$ has the cancellation property, $B \cong D$, so $E / J$ has the internal cancellation property. As we previously remarked, this is equivalent to the statement that $E / J$ is unit regular.

CoRollary 2.6. If $M$ is a quasi-injective module and $E$ its endomorphism ring, then the following properties are equivalent: (i) $M$ has the cancellation property, (ii) $M$ has the substitution property, (iii) $M$ has the internal cancellation property, (iv) $E / J(E)$ is unit regular, (v) $M$ is not isomorphic to a proper summand of itself.

Proof. If $M$ is quasi-injective, it is well known (see for example $[11,2.18$ and 2.21]) that $E / J(E)$ is von Neumann regular and that idempotents modulo $J(E)$ can be lifted. This and the previous theorem imply the equivalence of (i), (ii), (iii), and (iv), none of which depend on any other property of quasi-injective modules. (iii) trivially implies (v). (v) implies (i) for quasi-injective modules by $[11,6.20]$.

3. Stable range theorems for endomorphism rings. A number of theorems are known which assert that certain rings have certain integers in the stable range. In [3], Bass showed that if $S$ is a commutative $J$-Noetherian ring of $J$-dimension $d$ and $R$ is a finite $S$-algebra then $d+1$ is in the stable range for $R$. When $S$ is not Noetherian the endomorphism ring of a finitely generated $S$-module (or $R$-module) need not be finite over $S$, so we need a new result. In this section, Bass's result is extended to the endomorphism rings of finitely presented $R$-modules. The case of finitely generated modules remains open. Other recent results on stable range are Heitman's theorem [15] that a commutative ring with (classical) Krull dimension $d$ has $d+2$ in the stable range $(d+1$ if it is a domain) and Stafford's theorem [28] that ideal invariant Noetherian rings of (noncommutative) Krull dimension $d$ have $d+1$ in the stable range. Both of these are promising, but do not apply to many endomorphism rings.

LeMma 3.1. Let $A$ be a cyclic semi-simple module over a ring $R$ and $x_{1}, x_{2}$ elements of $A$ such that $A=x_{1} R+x_{2} R$. Then there is an $r \in R$ such that for all central units $u \in R, A=\left(x_{1}+x_{2} u r\right) R$.

REMARK. This is a slight generalization of a lemma of Swan's [30, Lemma 4] which generalizes a lemma due to Bass. Swan's lemma would suffice for this section, but we will need the more 
general result in the next section.

Proof. There is a submodule $B \subseteq x_{2} R$ such that $A=x_{1} R \oplus B$. We first find an element $b \in B$, such that $A=\left(x_{1}+b\right) R$. Proceeding by induction on the length of $B$, it suffices to show that we can find such a $b$ with $\alpha\left(x_{1}+b\right)>\alpha(x)$ (where $\alpha(x)$ is the right annihilator of $x)$. Since $\alpha\left(x_{1}+b\right)=\alpha\left(x_{1}\right) \cap \alpha(b)$, this will be possible unless $\alpha\left(x_{1}\right) \subseteq \alpha(B)$, where $\alpha(B)$ is a two-sided ideal. If this happens, we will show $B=0$. Since $A \alpha(B) \subseteq x_{1} R$, we may pass to $A / A \alpha(B)$ and, in effect, assume $\alpha(B)=0$. In this case, $\alpha\left(x_{1}\right)=0$, so $x_{1} R \cong R$. Hence our ring $R$ is actually semi-simple Artinian, and $A \cong R_{R} \oplus B$, which, since $A$ is cyclic, clearly implies $B=0$.

Finally, if $u$ is a central unit of $R$, then multiplication by $u$ gives an automorphism of any $R$-module, so the map of $A$ into itself taking $r x_{1}+y(y \in B)$ to $r x_{1}+y u$ is an automorphism, and therefore takes the generator $x_{1}+b$ to another generator, $x_{1}+b u$. This completes the proof of 3.1 .

Lemma 3.2. A ring $E$ has $n$ in the stable range if and only if for every cyclic module $A$ and elements $a_{i} \in A, 1 \leqq i \leqq r$ where $r>n$, such that $A=a_{1} E+\cdots+a_{r} E$, there are elements $b_{i} \in E$, $1 \leqq i \leqq r-1$, such that $A=\left(a_{1}+a_{r} b_{1}\right) E+\cdots+\left(a_{r-1}+a_{r} b_{r-1}\right) E$.

Proof. This is essentially Bass's proof from [3, 4.1] that if $n$ is in the stable range for $E$ then it is in the stable range for homomorphic images of $E$. We assume that $A=E / I$, where $I$ is a right ideal, and that $n$ is in the stable range for $E$. If $a_{i}=c_{i}+I$ then there is an element $c_{r+1} \in I$ such that $\left(c_{1}, \cdots, c_{r}, c_{r+1}\right)$ is right unimodular. Since $n$ is in the stable range for $E$, there are elements $d_{i} \in E, 1 \leqq i \leqq r$ such that $\left(c_{1}+c_{r+1} d_{1}, \cdots, c_{r}+c_{r+1} d_{r}\right)$ is a right unimodular row. Since $I$ is a right ideal, $c_{i}+c_{r+1} d_{i}$ is in the same coset as $c_{i}$, so, in effect, we may assume that the original elements $c_{i}$ form a right unimodular row. The result now follows immediately, by again applying the stable range condition.

LEMMA 3.3. Let $S$ be a commutative ring, $s \in S$, and $T=\{t \in S$ : for some $x$ and $y$ in $S, x t+y s=1$. Then if $\phi: S \rightarrow T^{-1} S$ is the natural map, $\phi(s) \in J\left(T^{-1} S\right)$, and if $f: S \rightarrow S^{\prime}$ is any ring homomorphism such that $f(s) \in J\left(S^{\prime}\right)$, then $f$ factors uniquely through $\phi$. Furthermore, the correspondence $I \rightarrow \phi^{-1} I$ induces a one-to-one correspondence between the maximal ideals of $T^{-1} S$ and those maximal ideals of $S$ which contain s.

Proof. Note that if $t \in T$ and $x q+y s=t$ in $S$, then $q \in T$ (since 
if $a t+b s=1$ then $(a x) q+(a y+b) s=1)$. If $a / b \in T^{-1} S(b \in T)$ then to show $1-(a / b) \phi(s)$ is a unit, we show that $b-a s \in T$, which is clear, since $(b-a s)+a s=b$ and $b \in T$ (using our previous remark). This shows that $\phi(s) \in J\left(T^{-1} S\right)$. If $f: S \rightarrow S^{\prime}$ is a ring homomorphism and $f(s) \in J\left(S^{\prime}\right)$, and $x t+y s=1$, then $f(1-y s)$ is a unit, so $f(x t)$ is a unit, from which our universal property follows. It is clear that if $M$ is a maximal ideal of $S$ then either $s \in M$ or $M \cap T$ is nonempty, but never both. This shows that the maximal ideals of $S$ which correspond to maximal ideals of $T^{-1} S$ are precisely those which contain $s$. If $M$ is a maximal ideal of $T^{-1} S$, then $\phi(s) \in M$ (since $\phi(s) \in J\left(T^{-1} S\right)$ ) so $s \in \phi^{-1} M$. If $N$ is a maximal ideal of $S$ containing $\dot{\phi}^{-1} M$, then $s \in N$, so by our previous remark $T^{-1} N$ is a maximal ideal of $T^{-1} S$. Since $M=T^{-1} \phi^{-1} M$, it follows that $M \subseteq T^{-1} N$, and hence that $N=\phi^{-1} M$. Hence $\phi^{-1} M$ is maximal, which completes the proof of 3.3.

We recall that a $J$-ideal of a commutative ring is an ideal which is the intersection of maximal ideals, and that $J$-prime is a prime $J$-ideal. A commutative ring $S$ is $J$-Noetherian if it satisfies the ascending chain condition on $J$-ideals, in which case every $J$-ideal is the intersection of a finite number of $J$-primes. The $J$-dimension of $S$ is the maximum of the lengths of chains of $J$-primes, and the $J$-height of a $J$-prime $P$ is the maximum of the lengths of chains of $J$-primes contained in $P$. The following result generalizes Bass's theorem in [3] that if $S$ is a commutative $J$-Noetherian ring of $J$-dimension $d$ and $R$ is a finite $S$-algebra, then $d+1$ is in the stable range for $R$. The main difference of technique between the proof that follows and previous proofs of related results is that we are forced to use induction on a sequence of localizations rather than on homomorphic images.

THEOREM 3.4. Let $S$ be a commutative $J$-Noetherian ring of $J$-dimension $d$ and $R$ an S-algebra such that for all J-primes $P$ of $S, R_{P}$ is a finite $S_{P}$-algebra. Let $A$ be a finitely presented $R$-module and $E$ the endomorphism ring of $A$. Then $d+1$ is in the stable range for $E$.

Proof. Let $\left(a_{1}, \cdots, a_{r}\right)$ be a right unimodular row $(r>n)$ and $L$ the set of elements of $S$ not contained in any minimal $J$-prime (i.e., $J$-prime of height zero). The maximal ideals of $L^{-1} S$ are precisely the ideals extended from the minimal $J$-primes of $S$ (by [31, Lemma 12.9 and Prop. 5.5] or [22, Thms. 34 and 81]), from which it follows that $\left(L^{-1} S\right)$ has only a finite number of maximal ideals. Since $A$ is finitely presented,

$$
L^{-1} E=\operatorname{End}_{L^{-1} R}\left(L^{-1} A\right) .
$$


It follows from 2.3 that $L^{-1} E$ is Artinian modulo its radical. Applying Lemma 3.1 , we obtain an element $w \in a_{2} L^{-1} E+\cdots+a_{r} L^{-1} E$ such that for every $\lambda \in L, a_{1}+\lambda w$ is a unit in $L^{-1} E$. Choosing a suitable $\lambda$, we may assume that the coefficients of $w$ are all in $E$, and if $\left(a_{1}+w\right) v=1$ then $v=u / s$ for some $s \in L$, so we may assume that $w \in a_{2} E+\cdots+a_{r} E$ and $\left(a_{1}+w\right) E \supseteqq s E$.

We now make a standard remark that is generally useful in arguments of this sort: that we can without loss replace $a_{1}$ by $a_{1}+w$, if $w \in a_{2} E+\cdots+a_{r} E$. Clearly, if $\left(a_{1}, \cdots, a_{r}\right)$ is right unimodular, then so is $\left(a_{1}+w, a_{2}, \cdots, a_{r}\right)$. If $\left(a_{1}+w\right) x_{1}+a_{2} x_{2}+\cdots+$ $a_{r} x_{r}=1$ and $\left(x_{1}, \cdots, x_{r-1}\right)$ is left unimodular, and $w=a_{2} w_{2}+\cdots+a_{r} w_{r}$ then $a_{1} x_{1}+a_{2}\left(x_{2}+w_{2} x_{1}\right)+\cdots+a_{r}\left(x_{r}+w_{r} x_{1}\right)=1$ and $\left(x_{1}, x_{2}+w_{2} x_{1}, \cdots\right.$ $\left.x_{r-1}+w_{r-1} x_{1}\right)$ is again left unimodular. In the particular situation we have here, this means that without loss of generality, we may assume that $a_{1} E \supseteqq s E$ for some $s \in L$.

We now let $T=\{t \in S$ : for some $x$ and $y$ in $S, t x+s y=1\}$. An examination of Lemma 3.3 shows that $s \in J\left(T^{-1} S\right)$, and that the lattice of $J$-primes of $T^{-1} S$ is on one-to-one correspondence with a sublattice of the $J$-primes of $S$ which does not contain any of the primes of $J$-height zero. It follows that $T^{-1} S$ is again $J$-Noetherian and of smaller $J$-dimension than the $J$-dimension of $S$. Using finite presentation again, we see that $T^{-1} E=\operatorname{End}_{T^{-1} R}\left(T^{-1} A\right)$, so we may use induction on the $J$-dimension to infer that $d$ is in the stable range for $T^{-1} E$. Now $E / s E=T^{-1}(E / s E)$, since every element of $T$ acts as a unit on $E / s E$ (from the definition of $T$ ). Hence $E / s E=$ $T^{-1} E / s T^{-1} E$, so $E / s E$ is a homomorphic image of $T^{-1} E$, and hence has $d$ in the stable range (by 3.2 or $[3,4.1]$ ). Since $a_{1} E \supseteqq s E$, $E / a_{1} E$ is a cyclic module over a ring with $d$ in the stable range. Using 3.2 again, we can find elements $b_{i}$, $(1<i<r)$ of $E$ such that $E / a_{1} E$ is generated by the images of the elements $a_{i}+a_{r} b_{i}$, $1<i<r$. It follows that $\left(a_{1}, a_{2}+a_{r} b_{2}, \cdots, a_{r-1}+a_{r} b_{r-1}\right)$ is a right unimodular row, which completes the proof of the theorem. (Note that the apparent asymmetry $b_{1}=0$ is produced by the fact that we started out by altering $a_{1}$.)

If the ring $S$ is a domain and the module in question is torsionfree, then the finite presentation requirement can be relaxed. We note first a general lemma.

Lemma 3.5. Let $A$ be a finitely generated module over an algebra $R$ over a commutative ring $S, T$ a multiplicatively closed subset of $S$, and $B$ a finitely generated $R$-module such that if $x \in B$ and $x t=0$ for some $t \in T$ then $x=0$. Then the natural map

$$
T^{-1} \operatorname{Hom}(A, B) \Longrightarrow \operatorname{Hom}\left(T^{-1} A, T^{-1} B\right)
$$


is an isomorphism.

Proof. If $\phi$ is the natural map and $\phi(f)=0$ then $f(A) \subseteq\{x \in B$ : for some $t \in T, t x=0\}$. By hypothesis, $f=0$ so $\phi$ is injective. Let $g \in \operatorname{Hom}\left(T^{-1} A, T^{-1} B\right)$ and let $\left\{x_{1}, \cdots, x_{k}\right\}$ be a set of generators for $A$. If $g\left(x_{i}\right)=y_{i} / t_{i}$ (with $y_{i} \in B, t_{i} \in T$ ) and $t$ is the product of $t_{i}$, then $(t g)(A) \subseteq \psi(B)$, where $\psi: B \rightarrow T^{-1} B$ is the natural map (which, in this case, is injective). If $h: A \rightarrow B$ is defined by $h(x)=t g(x)$, then clearly $g=\phi(h / t)$, so $g \in \phi\left(T^{-1} \operatorname{Hom}(A, B)\right)$. This shows that $\phi$ is surjective.

THEOREM 3.6. Let $S$ be a commutative J-Noetherian domain of $J$-dimension $d$ and $R$ an S-algebra such that for all J-primes $P$ of $S, R_{P}$ is a finite $S_{P}$-algebra. Let $A$ be a finitely generated $R$-module which is torsion-free as an $S$-module, and $E$ the endomorphism ring of $A$. Then $d+1$ is in the stable range for $E$.

Proof. The proof is the same as that of 3.4, using $S$-torsionfreeness and Lemma 3.5 instead of finite presentation.

This is the most general result known to the author about the stable range of endomorphism rings. Some more specialized results concerning endomorphism rings with two in the stable range are in the following two sections. Stafford's previously mentioned stable range theorem [28] includes, in particular, fully bounded Noetherian rings, and we present a generalization of this which also includes the classical examples of finite algebras over commutative $J$-Noetherian rings (but, as far as we can tell, does not include the result of 3.4). We need first a lemma to replace 3.1. The following lemma was obtained independently by the author [39] and Stafford [28].

LEMma 3.7. Let $R$ be a semi-prime right Goldie ring, $a \in R$, and $I$ a right ideal such that $a R+I$ is an essential right ideal. Then there is $a b \in I$ such that $(a+b) R$ is essential.

THEOREM 3.8. Let $R$ be a ring and $J$ an ideal contained in the Jacobson radical of $R$ such that $R / J$ is a semiprime right Goldie ring. Assume that $R / J$ is bounded (that is, every essential right ideal contains an essential ideal), and that for every essential ideal $I$ of $R / J,(R / J) / I$ has $d$ in the stable range, where $d$ is some integer, $d \geqq 1$. Then $R$ has $d+1$ in the stable range.

Proof. We may assume $J=0$. If $\left(a_{1}, \cdots, a_{r}\right)$ is a right unimodular row, then by 3.7 there is a $w \in a_{2} R+\cdots+a_{r} R$ such that 
$\left(a_{1}+w\right) R$ is essential. By the argument in the second paragraph of the proof of 3.4, we may replace $a_{1}$ by $a_{1}+w$, and assume that $a_{1} R$ is essential. In this case, $a_{1} R$ contains an essential ideal $I$, and $R / a_{1} R$ may be regarded as a cyclic $R / I$-module. Just as in the proof of 3.4, an application of Lemma 3.2 completes the proof.

In a mild extension of the previous theory to the noncommutative case, we will consider rings $R$ such that for every simple module $S, R / \alpha(S)$ is Artinian, where $\alpha(S)$ is the annihilator of $S$. In such a ring, the radical is the intersection of the maximal twosided ideals. We will call an ideal in such a ring $R$ a $J$-ideal if it is an intersection of maximal (two-sided) ideals. A $J$-prime is a prime ideal which is a $J$-ideal, and a ring is $J$-Noetherian if it satisfies the ascending chain condition on $J$-ideals. $R$ has $J$-dimension $n$ if every chain of $J$-primes has at most $n+1$ elements, and $n$ is the smallest integer for which this is true. We call an ideal $I$ in $R$ a Goldie ideal if $R / I$ is a right Goldie ring, and we call $I$ semiprime if it is the intersection of prime ideals. In particular, every $J$-ideal is semiprime. We will need the following lemma from [26, 1.3 and 1.4]:

Lemмa 3.9. If $S$ is a right Goldie semiprime ideal, then $S$ is the intersection of a finite number of Goldie prime ideals. If $S=$ $P_{1} \cap \cdots \cap P_{n}=Q_{1} \cap \cdots \cap Q_{m}$ where the $P_{i}$ and $Q_{j}$ are Goldie prime ideals such that if $P_{i} \subseteq P_{j}$ or $Q_{i} \subseteq Q_{j}$ then $i=j$, then $m=n$ and, after rearrangement, $P_{i}=Q_{i}, i=1, \cdots, n$. The $P_{i}$ that appear in such a representation are precisely the primes minimal over $S$. Conversely, if $\left\{P_{1}, \cdots, P_{n}\right\}$ is a set of right Goldie primes then $S=$ $P_{1} \cap \cdots \cap P_{n}$ is a right Goldie semiprime ideal. Hence the set of right Goldie semiprime ideals is in one-to-one correspondence with the set of finite sets of mutally incomparable right Goldie primes.

THEOREM 3.10. Let $R$ be a ring such that for every simple module $S, R / \alpha(S)$ is Artinian, and such that $R$ is J-Noetherian and of J-dimension d. Suppose in addition that for each J-prime $P$, $R / P$ is right Goldie and bounded. Then $d+1$ is in the stable range for $R$.

Proof. We first need to note that every $J$-ideal of $R$ is a finite intersection of $J$-primes. This is standard in the commutative case, and the proof here is similar, but we will give it for completeness. (It is lifted from the proof of [22, Thm. 87]). If the result were false, then since $R$ is $J$-Noetherian, there would be a $J$-ideal $I$ maximal with respect to the property that it is not such an intersection. Since $I$ can not be prime, there are strictly larger ideals $A$ 
and $B$ with $A B \cong I$. For any ideal $X$, we let $J-\operatorname{rad}(X)$ be the intersection of the maximal ideals containing $X$. If $K=J-\operatorname{rad}(A)$ and $L=J-\operatorname{rad}(B)$, then $K$ and $L$ are $J$-ideals larger than $I$, and hence are finite intersections of $J$-primes, so it will suffice to prove that $K \cap L=I$. If not, since $I$ is a $J$-ideal, there is some maximal ideal $M$ which contains $I$ but does not contain $J \cap K$, and hence contains neither $A$ nor $B$. In this case, $R=M+A=M+B$, so $R=R^{2}=$ $M^{2}+A M+M B+A B$, and since $A B \subseteq I \subseteq M, R^{2} \subseteq M$, which is impossible.

From 3.9, it follows that every $J$-ideal $I$ of $R$ is right Goldie, and the hypotheses of the theorem also imply that $R / I$ is bounded. (If $I=P_{1} \cap \cdots \cap P_{n}$, where the $P_{i}$ are minimal over $I$, and $Q$ and $Q_{i}$, $(i=1, \cdots, n)$ are the quotient rings of $R / I$ and $R / P_{i}$ respectively, then we can identify $Q=Q_{1} \times \cdots \times Q_{n}$. If $L$ is an essential left ideal of $R / I$, the $L \cap Q_{i}$ is essential in $R / P_{i}$, and hence contains an essential two-sided ideal in $R / P_{i}$, which can be identified with an ideal $B_{i} \subseteq(R / I) \cap Q_{i}$. The ideal $B=B_{1} \times \cdots \times B_{n}$ is the desired bound.)

We now prove 3.10 by induction on $d$. As usual we may assume that Jacobson radical is zero, which, by the previous argument, shows that we may assume that $R$ is a bounded semiprime right Goldie ring. Since an essential two-sided ideal is not contained in any minimal $J$-prime (using 3.9 mildly again), we see that any two-sided essential ideal $I, R / I$ satisfies all of the hypotheses of the theorem and its $J$-dimension is one less. An application of 3.9 and the induction hypothesis completes the proof.

\section{Special results for Krull dimension one.}

THEOREM 4.1. Let $S$ be a commutative ring of Krull dimension one with a finite number of minimal primes, and $R$ an S-algebra such that for every prime $P$ of $S, R_{P}$ is a finite $S_{P}$-algebra. Let $A$ be a finitely generated $R$-module and assume that either $A$ is finitely presented or that $S$ is a domain and $A$ is S-torsion-free. Then 2 is in the stable range for $\operatorname{End}(A)$.

Proof. Let $E=\operatorname{End}(A)$. If $L$ is the set of elements of $S$ not in any minimal prime then either hypothesis implies that $L^{-1} E=$ $\operatorname{End}_{L^{-1} R}\left(L^{-1} A\right)$. By 2.3, $L^{-1} E$ is Artinian modulo its radical, and has 1 in the stable range. Following the proof of 3.4, we see that it will suffice to show that if $s \in L$, and $T=\{t \in S$ : for some $x$ and $y$ in $S, t x+s y=1\}$, then one is in the stable range for $T^{-1} E$. From 3.3 it is clear that $T^{-1} S / J\left(T^{-1} S\right)$ is zero dimensional, and the hypo- 
theses imply that $T^{-1} E=\operatorname{End}_{T^{-1} R}\left(T^{-1} A\right)$, so by the proof of [13, Theorem 18], it follows that one is in the stable range for $T^{-1} E$, as desired.

We now remind the reader that there is a theory of Krull dimension for noncommutative rings which agrees with the usual Krull dimension for commutative Noetherian rings, but not, in general, for other commutative rings. For details we refer to [14]. In particular, a ring $R$ has (noncommutative) Krull dimension at most one if for every descending chain of right ideals $I_{1} \supseteqq I_{2} \supseteqq \cdots \supseteqq I_{n} \supseteqq \cdots$, all but a finite number of the factors $I_{j} / I_{j+1}$ are Artinian.

THEOREM 4.2. If $R$ is a ring with (noncommutative) Krull dimension at most one, than 2 is in the stable range for $R$.

Proof. As usual we may assume $J(R)=0$. By $[14,3.4] R$ is then a semiprime right Goldie ring, so that if $a x+b y+c z=1$, we may use 3.7 and the argument in paragraph two of the proof of 3.4 and assume that $a R$ is essential in $R_{R}$. By $[14,6.1]$ since $R$ is semiprime, this implies that $R / a R$ has Krull dimension zero and hence is Artinian. It will therefore suffice to show that if $A$ is a cyclic Artinian module over any ring $R$ and $A=b R+c R$ then for some $r \in R, A=(a+b r) R$. If $K$ is the intersection of the maximal submodules of $A$, then it is easy to verify that $A / K$ is semi-simple and that it will suffice to find an $r$ such that $A=K+(a+b r) A$. We therefore may assume that $K=0$, in which case our result is just 3.1.

COROLlaRY 4.3. If $R$ is a ring of (noncommutative) Krull dimension one and $P$ is a finitely generated projective $R$-module, then one is in the stable range for $\operatorname{End}(P)$. In particular, if $P \oplus P \oplus P \oplus X \cong P \oplus Y$ then $Y \cong P \oplus P \oplus X$ and if $P \oplus P \oplus X \cong$ $P \oplus Z$ then $Z \cong P^{\prime} \oplus X$ where $P \oplus P^{\prime} \cong P \oplus P$.

Proof. This follows from the results of section one and [14, 1.2], which shows that $\operatorname{End}(P)$ has Krull dimension at most one. Swan's example [29] shows that there can arise modules $P^{\prime}$ in the above situation which are not isomorphic to $P$.

J.C. Robson has pointed out that the above result can be used to provide an alternative proof of Stafford's theorem in [28] that if $R$ is a Noetherian domain and for some integer $k, M_{k}(R)$ is a principal left and right ideal ring, then $M_{n}(R)$ is a principal left and right ideal ring for all $n \geqq 2$. We obtain an expanded result (which could also be obtained easily from Stafford's argument). Here $M_{k}(R)$ is the $k \times k$ matrix ring over $R$, and we use the term "Dede- 
kind ring" in the sense of Robson $[27,6]$. For information on principal ideal rings, we refer to [18]. We recall that if $R$ is a left principal ideal ring and right Noetherian, then $R$ is the product of an Artinian ring and prime rings. Since primeness is a Morita invariant, we restrict attention to matrix rings over prime rings.

THEOREM 4.4. If $R$ is a Noetherian prime ring, then the following properties of $R$ are equivalent:

(i) for some integer $k, M_{k}(R)$ is a principal left and right ideal ring;

(ii) $R$ is Morita equivalent to a principal left and right ideal ring;

(iii) either (a) $R$ is a principal left and right ideal ring, or (b) $R$ is a domain and $M_{k}(R)$ is a principal left and right ideal ring for all $k \geqq 2$;

(iv) $R$ is a Dedekind prime ring and for every essential right $I$ there is a finitely generated free module $F$ such that $F \oplus I$ is free.

Proof. Each of the conditions implies that $R$ is a Dedekind prime ring. Every Dedekind prime ring is Morita equivalent to a Dedekind domain, so we let $D$ be this domain. Every projective $D$-module is the direct sum of a free module and a right ideal [6]. Now if $D$ is Morita equivalent to a principal left and right ideal ring, then there is some finitely generated projective module $P$ such that every submodule of $P$ is a homomorphic image of $P$. If $Q$ is projective of the same rank as $P$, then $Q$ is a homomorphic image of $P$ only if they are isomorphic. Hence, if $k$ is the rank of $P$, then $R^{k} \cong R^{k-1} \oplus I$ for every nonzero right ideal $I$. 4.3 implies immediately that $R^{2} \cong R \oplus I$, so that every projective $D$-module is either free or isomorphic to a right ideal. This immediately implies that for all $n \geqq 2, M_{n}(R)$ is a principal ideal ring, and also that every ring Morita equivalent to $D$ is either a domain or a matrix ring over $D$. This shows the equivalence of (i), (ii) and (iii) in Theorem 4.4. Turning to (iv), given 4.3 this means that for every essential right ideal $I, R \oplus I \cong R^{2}$, so (iv) implies (i), and (i) trivially implies (iv). This completes the proof of the theorem.

Remark. From [40] we know that the Weyl algebra $A_{1}$ is an example of a simple Dedekind ring which is not a principal left and right ideal ring but which has the property that $M_{2}\left(A_{1}\right)$ is a principal left and right ideal ring. An example which is an order over a commutative Dedekind domain, is in [29]. In the proof of Theorem 3 of [29], Swan constructs a maximal order $A$ in a quater- 
nion algebra, with a left ideal $P$ such that $P \oplus \Lambda \cong \Lambda \oplus \Lambda$ and $P$ is not principal. In addition, he shows that the class number of $A$ is 2 , so that every nonzero left ideal of $\Lambda$ is either principal or isomorphic to $P$. It is clear from this that $M_{2}(\Lambda)$ is a principal left and right ideal ring. Examples of algebras in characteristic $p$ with this property have recently been discovered by V. Jategaonkar [19].

5. Cancellation theorems for torsion-free Abelian groups, Modules over valuation rings, and complex tori.

LEMMA 5.1. Let $M$ be a torsion-free module of finite rank over an integral domain $R$ and let $f: M \rightarrow M$ be an injective endomorphism. Then there is an $r \in R, r \neq 0$, such that $r M \subseteq f(M)$.

REMARK. This was proved for $R=Z$ by Jónsson in [20]. I do not know who first gave a proof valid in this generality. The following argument is due to E. A. Walker. Note that in the Abelian group case, the lemma says that a subgroup of $M$ isomorphic to $M$ has finite index.

Proof. Let $K$ be the quotient field of $R$ and regard $f$ as an endomorphism of $K \otimes M$. By the Cayley-Hamilton theorem, $f$ is a root of its characteristic polynomial, so we have an equation

$$
f^{n}+r_{n-1} f^{n-1}+\cdots+r_{1} f=-\operatorname{det}(f) .
$$

Since each $r_{i}$ is a fraction, we can let $r$ be the product of the denominators times $-\operatorname{det}(f)$, and we see that for every $x \in M, r x \in$ $f(M)$, as desired. (Since $f$ is injective, $\operatorname{det}(f) \neq 0$, so $r \neq 0$.)

THEOREM 5.2. Let $R$ be a principal ideal domain, let $E$ be an $R$-algebra which is torsion-free and of finite rank over $R$, and let $J$ be the radical of $E$. Assume that $E=p E$ for all but a finite number of primes $p$ of $R$. Then $E / J$ is an Artinian ring.

Proof. Let $K$ be the quotient field of $R . \quad E \otimes K$ is a finite dimensional $K$-algebra, with nilpotent radical $N$. It is easy to see that $N \cap E$ is a nilpotent ideal of $E$, and $E /(N \cap E)$ is an order in $(E \otimes K) / N$, which is an Artinian semi-simple ring. It suffices to prove the result for $E /(N \cap E)$, so we will assume $N=0$.

If $D$ is the maximal divisible $R$-submodule of $E$, then $D$ is an ideal of $E$, since it is a fully-invariant $R$-submodule. Since $D$ can be identified with $D \otimes K, D$ is actually an ideal, and hence a summand, of the ring $E \otimes K$. We therefore have a ring decomposition $E=D \oplus E^{\prime}$, where $D$ is semi-simple Artinian, and $E^{\prime}$ is a torsion- 
free $R$-algebra with no divisible $R$-submodules other than 0 . Clearly, $J \subseteq E^{\prime}$, and it will suffice to prove that $E^{\prime} / J$ is Artinian. Let $m$ be the product of the prime elements of $R$ which do not divide $E$. Since $R$ is a principal ideal domain, if $E^{\prime}$ has rank $k$, then every finitely generated $R$-submodule of $E^{\prime}$ can be generated by $k$ elements, so $E^{\prime} / m E^{\prime}$ is a finitely generated, Artinian $R$-module. (If $R=Z, E^{\prime} / m E^{\prime}$ is finite of order at most $m^{k}$.) The result will therefore be proved if we show that $J \supseteqq m E^{\prime}$.

If $x \in m E^{\prime}$ then $1-x$ includes (by right multiplication) the identity map on $E^{\prime} / m E^{\prime}$. We show that right multiplication by $1-x$ is an $R$-automorphism of $E^{\prime}$. For convenience, we denote this $R$-endomorphism by $f$. If $y \in \operatorname{ker}(f)$, and $y \neq 0$, then there is a $y^{\prime} \in E^{\prime}$ and an integer $n$ such that $n y^{\prime}=y$ and $y^{\prime} \notin m E^{\prime}$ (since, otherwise, the $R$-submodule $K y^{\prime} \cap E^{\prime}$ would be divisible). Since $E^{\prime}$ is torsion-free, $y^{\prime} \in \operatorname{ker}(f)$, which would imply that the induced map on $E^{\prime} / m E^{\prime}$ was not one-to-one. This implies that $f$ is injective, so we must show $f\left(E^{\prime}\right)=E^{\prime}$. Since for every prime $p$ not dividing $E^{\prime}$ we have $E^{\prime}=p E^{\prime}+f\left(E^{\prime}\right)$, by hypothesis, we conclude that $E^{\prime} / f\left(E^{\prime}\right)$ is a divisible module. By the previous lemma, there is a nonzero $r \in R$ such that $r\left(E^{\prime} \mid f\left(E^{\prime}\right)\right)=0$. The only divisible module with this property is 0 , so $E^{\prime}=f\left(E^{\prime}\right)$, as desired.

Corollary 5.3. If $A, B$, and $C$ are Abelian groups, $A$ is torsion-free of finite rank, and $A \oplus B \cong A \oplus C$, and if there is a finite set $\pi$ of primes such that either (i) $A=p A$ for all primes $p, p \notin \pi$, or (ii) $B=p B$ for all primes $p, p \notin \pi$, then $B \cong C$.

Proof. In case (i), the endomorphism ring of $A$ is a ring of the sort described in Theorem 5.2, so 2.1 and 2.2 prove the result. We prove case (ii) by reducing it to case (i). We first show that in case (ii), $C=p C$ for all primes $p, p \notin \pi$. Since $(A \oplus C) / p(A \oplus C) \cong$ $A / p A \oplus C / p C$, and $(A \oplus B) / p(A \oplus B) \cong A / p A$, wesee that either $C=p C$ or $A / p A$ is isomorphic to a proper summand of itself, which is impossible since it is finite. We next let $B^{\prime}$ and $C^{\prime}$ be the subgroups of $B$ and $C$ consisting of elements of finite order prime to the elements of $\pi . \quad B^{\prime}$ and $C^{\prime}$ are torsion divisible groups, and thus summands of $B$ and $C$. Since $A$ is torsion-free, $B^{\prime}$ and $C^{\prime}$ are precisely the subgroups of $A \oplus B$ and $A \oplus C$ consisting of elements of finite order prime to the elements of $\pi$, so $B^{\prime} \cong C^{\prime}$, and if $B=$ $B^{\prime} \oplus B^{\prime \prime}$ and $C=C^{\prime} \oplus C^{\prime \prime}$, we have $A \oplus C^{\prime \prime} \cong A \oplus B^{\prime \prime}$. We may assume, therefore, that $B^{\prime}=C^{\prime}=0$. In this case, if we localize at $\pi$, we have $B_{\pi} \cong B$ and $C_{\pi} \cong C$. Since $A_{\pi} \oplus B_{\pi} \cong A_{\pi} \oplus C_{\pi}$, and $A_{\pi}$ is torsion-free of finite rank, and is divisible by all primes not in $\pi$, we conclude from case (i) that $B_{\pi} \cong C_{\pi}$, whence $B \cong C$. 
Case (ii) above, in the special case in which $B$ and $C$ also have finite rank, was obtained by Lady in [24].

A number of generalizations of the above results and of 5.6 below are possible to torsion-free modules over special classes of domains. We mention only the following, for a non-Noetherian valuation ring, by which we mean a commutative domain whose ideal lattice is a chain.

THEOREM 5.4. If $R$ is a valuation ring and $E$ a torsion-free $R$-algebra of finite rank, then $E / J(E)$ is Artinian. In particular, every torsion-free $R$-module of finite rank has the cancellation property.

Proof. If $E / J(E)$ is not Artinian, then $E$ has homomorphic images which are semi-simple $E$-modules of arbitrary finite length. Now if $A$ is a finitely generated $R$-submodule of $E$, then since $R$ is a valuation ring, $A$ is a free module of rank at most equal to the rank of $E$ as an $R$-module. In particular, if $M$ is the maximal ideal of $R$, then the $R / M$-dimension of $A / M A$ is bounded by the $R$-rank of $E$. This property is inherited by homomorphic images, so if $k$ is the rank of $E$, and $N$ is a $R$-homomorphic image of $E$, and $B$ is a finitely generated $R$-submodule of $N$, then $B / M B$ has rank at most $k$. This implies that in any direct sum decomposition of $N$ as an $R$-module there are at most $k$ summands. It follows that there is a bound on the length of cyclic, semi-simple $E$-modules, which, by our original remark, implies that $E / J(E)$ is Artinian.

THEOREM 5.5. If $A$ is a torsion-free Abelian group of rank one, and $A$ is not isomorphic to $Z$, then $A$ has the cancellation property if and only if it has the substitution property.

Proof. Fuchs and Loonstra show in [10] that such a group $A$ has the cancellation property if and only if for every positive integer $n$, every automorphism of $A / n A$ lifts to an automorphism of $A$. If $E$ is the endomorphism ring of $A$, then there is an induced ring homomorphism $E \rightarrow \operatorname{End}(A / n A)$ whose kernel is easily seen to be $n E$. The map $E \rightarrow \operatorname{End}(A / n A)$ is surjective since every endomorphism of the cyclic group $A / n A$ is given by multiplication by some integer. Hence, if $A$ has the cancellation property, then for every integer $n$, every unit in $E / n E$ comes from a unit of $E$. If $a x+b y=1$ in $E$ then modulo $b E, a x=1$, so $a$ is a unit modulo $b$, and the condition says that for some $t \in E, a+b t$ is a unit in $E$. If $a+b t=u$ then $a u^{-1}+b t u^{-1}=1$, so 1 is in the stable range for $E$, and $A$ has the substitution property. 
We remark that examples in [7] show that there are subrings $E$ of the ring of rational numbers which are not Artinian modulo their radicals and which do have 1 in the stable range. These can be used to give a variety of examples of rank one groups with the cancellation property.

Theorem 5.6. If $A$ is a torsion-free Abelian group of finite rank, then 2 is in the stable range for $\operatorname{End}(A)$, and if $X$ is an Abelian group and $A \oplus A \oplus X \cong A \oplus Y$, then $Y \cong X \oplus A^{\prime}$, where $A^{\prime}$ is a group such that $A \oplus A \cong A \oplus A^{\prime}$.

Proof. It suffices to show that 2 is in the stable range for any $Z$-algebra which is torsion-free of finite rank. As in the proof of 5.2 we see that such a ring $E$ has a nilpotent ideal $N$ such that $E / N$ is a torsion-free $Z$-algebra which is semiprime. We may assume $N=0$, so that $E$ is semiprime. If $I$ is an essential left ideal of $E$ then $I$ contains a regular element, so $I$ contains a left ideal isomorphic (as a left module) to $E$. Lemma 5.1 implies that there is an integer $n, n \neq 0$, with $n E \subseteq I . \quad n E$ is an ideal and $E / n E$ is finite. Since finite rings have one in the stable range (2.2), $3.8 \mathrm{im}$ plies that $E$ has 2 in the stable range.

REMARK. The smallest group $A$ known to the author for which such an $A^{\prime}$ exists which is not isomorphic to $A$ has rank 16. (The existence of such a group follows from Swan's example [29] of a ring $\Lambda$ which is free Abelian of rank 16 with a projective module $P$ such that $P \oplus \Lambda \cong \Lambda \oplus \Lambda$ as left modules, with $P \not \Lambda$. Find the Abelian group $A$ by applying Zassenhaus's existence theorem [41], and use the correspondence (as in [2]) between projective $\Lambda$-modules and summands of direct sums of copies of $A$ to get the example).

In particular, if $A$ has rank one, then $\operatorname{End}(A)$ is a principal ideal ring, so $A \oplus A \cong A \oplus A^{\prime}$ implies $A \cong A^{\prime}$. Combining our result with a special case of Theorem 2 of [2], we see that if $A$ is a torsion-free Abelian group of rank one, and $X$ and $Y$ are nonisomorphic torsion-free Abelian groups with $A \oplus X \cong A \oplus Y$, then neither $X$ nor $Y$ has a summand isomorphic to $A$, but both must have subgroups of finite index which do have summands isomorphic to $A$.

For further results on cancellation and related questions for torsion-free Abelian groups of finite rank, it is convenient to localize the category of Abelian groups. We recall that two torsion-free Abelian groups of finite rank are quasi-isomorphic if each isomorphic to a subgroup of the other, or, equivalently (by 5.1) if one is isomorphic to a subgroup of finite index in the other. Walker 
points out in [36] that this is isomorphism in a new category obtained by taking the torsion-free groups as the objects but by taking for the morphisms the groups $[M, N]=Q \otimes \operatorname{Hom}_{z}(M, N)$. Formalizing this, we let $\mathscr{A}$ be the category of torsion-free groups of finite rank, and define $\mathscr{A}_{0}$ to be the category with the same objects as $\mathscr{A}$ and with morphisms $Q \oplus \operatorname{Hom}_{z}(M, N)$. Clearly, we could similarly localize at a prime ideal, defining $\mathscr{A}_{(p)}$. We give a general definition:

Definition. Let $\mathscr{\text { be }}$ an additive category, and $R$ a commutative ring such that there is an $R$-module structure on each of the morphism groups $\mathscr{A}(X, Y)$ and such that the composition map $\mathscr{A}(X, Y) \times \mathscr{A}(Y, Z) \rightarrow \mathscr{A}(X, Z)$ is $R$-bilinear. Then for any multiplicatively closed subset $S$ of $R$ not containing zero we define $S^{-1} \mathscr{A}$ to be category whose objects are the same as those of $\mathscr{A}$ and whose morphism groups are defined by

$$
\left(S^{-1} \mathscr{A}\right)(X, Y)=S^{-1}(\mathscr{A}(X, Y)) \text {. }
$$

We note that if $\mathscr{A}$ consists of finitely presented $R$-modules, then this corresponds to the usual localization of the objects, but otherwise the result is quite different. If $S$ consists of all elements of $R$ not in some prime ideal $P$, then, as usual, we denote $S^{-1} \mathscr{A}$ by $\mathscr{A}_{P}$.

Lemma 5.7. If $M$ and $N$ are torsion-free Abelian groups of finite rank, and $\mathscr{A}$ is the category of torsion-free Abelian groups of finite rank, then $M$ and $N$ are isomorphic in $\mathscr{L}_{(p)}$ (for a prime p) if and only if $M$ is isomorphic to a subgroup of $N$ of finite index prime to $p$.

Proof. If $f / s$ and $g / t$ are elements of $\mathscr{L}_{(p)}(M, N)$ and $\mathscr{A}_{(p)}(N, M)$ which are mutal inverses, then $f g \in \operatorname{End}(N)$ is just multiplication by $s t$ (using the fact that the $\operatorname{map} \operatorname{Hom}(M, N) \rightarrow \operatorname{Hom}(M, N)_{(p)}$ is injective). It follows that $f$ and $g$ are injective and that $M / g(N)$ and $N / f(M)$ are finite groups annihilated by $s t$, which proves the stated result.

Definition. Two torsion-free groups $M$ and $N$ of finite rank are of the same genus if they are isomorphic in $\mathscr{A}_{(p)}$ for all primes $p$.

LEMMA 5.8. If $M$ and $N$ are torsion-free groups of finite rank, $M$ and $N$ are of the same genus if and only if for every positive integer $n$, there is a subgroup $M^{\prime}$ of $N$ of index prime to $n$ such that $M \cong M^{\prime}$. 
Proof. Let $S$ be the set of integers relatively prime to $n$, and work in the category $S^{-1} \mathscr{A}$, where $\mathscr{C}$ is the category of Abelian groups. By the argument of Lemma 5.7, it will suffice to show that if $M$ and $N$ are in the same genus, then they are isomorphic in the category $S^{-1} \mathscr{A}$. Now if $X$ is an object in any additive category in which idempotents split, the functor taking $M$ to $\operatorname{Hom}(X, M)$ gives (as before) a category equivalence from the category of summands of finite direct sums of copies of $X$ to the category of finitely generated projective $\operatorname{End}(X)$-modules. We therefore let $E=$ $\operatorname{End}(M \oplus N)$, so that $S^{-1} E$ is the endomorphism ring of $M \oplus N$ in $S^{-1} \mathscr{A}$, and we must show that $\bar{M}=S^{-1} \operatorname{Hom}(M \oplus N, M)$ and $\bar{N}=$ $S^{-1} \operatorname{Hom}(M \oplus N, N)$ are isomorphic as $S^{-1} E$-modules. Since $S^{-1} E$ is an algebra over $S^{-1} Z$, which is Artinian modulo its radical, [13, Theorem 4] implies that two finitely presented $S^{-1} E$-modules are isomorphic if they are locally isomorphic-i.e., in this case if $\bar{M}_{(p)} \cong \bar{N}_{(p)}$ for all primes $p$ not contained in $S$. This follows immediately from the fact that $M$ and $N$ are isomorphic in $\mathscr{L}_{(p)}$.

REMARK. This shows that our notion of genus coincides with Lady's notion [24] of "near-isomorphism". We use the term genus to make clear the connection with other areas of algebra in which this term is standard, such as the theory of lattices over orders over Dedekind rings, and finitely generated nilpotent groups. The above proof uses ring-theoretic machinery which is more powerful than necessary, and a more elementary proof has been given by Arnold [1].

THEOREM 5.9. If $M$ and $N$ are torsion-free Abelian groups of finite rank, then $M$ and $N$ are of the same genus if and only if there is a positive integer $n$ such that $M^{n} \cong N^{n}$.

REMARK. This should be compared with Jacobinski's result [17, 2.8] which is the corresponding result for lattices over orders, and [38] which contains the corresponding result for finitely generated groups with finite commutator subgroups.

Proof. If $M^{n} \cong N^{n}$, then certainly $M^{n}$ and $N^{n}$ are isomorphic in $\mathscr{L}_{(p)}$. If $E=\operatorname{End}(M \oplus N)$, and we use the category equivalence between summands of direct sums of copies of $M \oplus N$ and projective $E$-modules, and $M$ and $N$ correspond to the projective modules $\bar{M}$ and $\bar{N}$, then what we have to prove is that if $\left(\bar{M}_{(p)}\right)^{n} \cong\left(\bar{N}_{(p)}\right)^{n}$ then $\bar{M}_{(p)} \cong \bar{N}_{(p)}$. This is what is usually called the " $n$th root property" for projective modules over the ring $E_{(p)}$. This follows directly from [13, Theorem 19], but can be argued directly as follows: two 
finitely generated projective modules over a ring $R$ with Jacobson radical $J$ are isomorphic if and only if they are isomorphic modulo $J$. If $J$ is the radical of $E_{(p)}$, then by 5.2 above, $E_{(p)} / J$ is Artinian. Finitely generated modules over an Artinian ring satisfy the $n$th root property because they satisfy a Krull Schmidt theorem.

We now turn to the converse. We first remark that if $X, Y$ and $W$ are finite rank torsion-free groups in the same genus, then $X$ is a summand of $Y \oplus W$, and, further, if $X \oplus X^{\prime} \cong Y \oplus W$, then $X^{\prime}$ is in the same genus as $X$. All of this follows from Lady's results [24].

We now define an equivalence relation on the isomorphism classes in the genus of a particular group $X$ by defining $Y$ and $W$ to be equivalent if for some positive integer $n, X^{n} \oplus Y \cong X^{n} \oplus W$. This is clearly an equivalence relation. We denote the equivalence class of $Y$ by $[Y]$. We define a group structure on these equivalence classes by defining $[Y]+[W]=[U]$ if $W \oplus Y \cong X \oplus U$. By Lady's result cited in the previous paragraph, such a $U$ exists, and any two such $U$ are clearly equivalent. The group so obtained is denoted $D_{x}$. For future reference, we note that we could equally well have defined $[U]$ by requiring only that for some positive integer $n, X^{n} \oplus W \oplus Y \cong X^{n+1} \oplus U$. It is clear that this operation is commutative and associative, that $[X]$ is an identity, and that the inverse of $[U]$ is $[V]$, where $U \oplus V \cong X \oplus X$ (again using Lady's result to show that such a $V$ exists). By Lady's theorem, every group in the genus is isomorphic to a summand of $X \oplus X$, so by [23] there are only a finite number of isomorphism types in the genus, so the group we have defined is a finite group. If $W$ is a group in the genus of $X$, one easily computes that the element $k[W]$ can be represented by $U$, where for some integer $n, X^{n} \oplus W^{k} \cong$ $X^{n+k-1} \oplus U$. In particular, if $k$ is the order of [W] in $D_{X}$, then $X^{n} \oplus W^{k} \cong X^{n+k}$. If $k \geqq 2$, the fact that 2 is in the stable range for $\operatorname{End}(X)$ implies that $W^{k} \cong X^{k}$. If $k=1$ then $[W]=[X]$ so $W \oplus X \cong X \oplus X$. Adding $W$ we obtain $W \oplus W \oplus X \cong W \oplus X \oplus X$ The original equation shows that the right hand side is isomorphic to $X^{3}$, so $W^{2} \oplus X=X^{2} \oplus X$. The fact that 2 is in the stable range for $\operatorname{End}(X)$ again applies, and shows that $W^{2} \cong X^{2}$. This completes the proof of the theorem.

COROLlaRY 5.10. If $X, Y$, and $W$ are torsion-free Abelian groups of finite rank and $X \oplus Y \cong X \oplus W$, then for some positive integer $n, Y^{n} \cong W^{n}$.

Proof. The hypothesis implies that $X \oplus Y$ and $X \oplus W$ are isomorphic in $\mathscr{A}_{(p)}$. By 5.2, 2.1 and 2.2, cancellation holds in $\mathscr{A}_{(p)}$ so 
$Y$ and $W$ are isomorphic in $\mathscr{A}_{(p)}$ for all primes $p$. They are therefore in the same genus, and the result follows from the previous theorem.

REMARK. This theorem and its corollary have been generalized slightly to modules over orders over certain Dedekind rings by Arnold in [1]. Starting from this result, Goodearl [12] studies a generalization of the stable range conditions of this paper, and concludes that in 5.10, the finite rank condition on $Y$ and $W$ can be dropped.

We recall that a complex torus of complex dimension $n$ is an analytic group of the form $C^{n} / L$ where $L$ is a lattice in $C^{n}$ and is therefore a free Abelian group of rank $2 n$. If $A=C^{n} / L$ and $B=$ $C^{m} / M$ then we recall [32, Thm.32] that $\operatorname{Hom}(A, B)$, the set of analytic group homomorphisms, is exactly the set of holomorphic maps preserving the identity, and can be identified with those elements of $\operatorname{Hom}_{z}(L, M)$ which extend to $C$-linear maps $C^{n} \rightarrow C^{m}$. It is easily verified that the category of complex tori is an additive category with cokernels in which idempotents split, and such that the homorphism groups are all finitely generated torsion-free groups. If $A$ is an object in an additive category in which idempotents split, and $E=\operatorname{End}(A)$, the functor taking $X$ to $\operatorname{Hom}(A, X)$ gives an equivalence of categories between the category of summands of finite direct sums of copies of $A$ and the category of finitely generated projective $E$-modules. Just as in the previous work in this section, given two complex tori $A$ and $B$, any question about whether $A$ is a factor of $B$ or of a product of copies of $B$ or whether for some integer $n, A^{n} \cong B^{n}$, becomes a question about projective modules over $\operatorname{End}(A \times B)$. To draw conclusions about these projective modules we can proceed in two ways. Since any finitely generated torsionfree $Z$-algebra is the endomorphism ring of a torsion-free group [40], the theory can be reduced to Abelian group theory. On the other hand, since we can factor out the nil radical without loss when dealing with projective modules, we can assume that $E \oplus Q$ is a semi-simple $Q$-algebra, thus reducing the theory to classical results on orders.

THEOREM 5.11. If $\mathscr{A}$ is the category of complex tori, the tori $A$ and $B$ are isomorphic in $\mathscr{A}_{(p)}$ (i.e., $A$ and $B$ are of the same genus) for all primes $p$ if and only if for every positive integer $n$, there is an isogeny $A \rightarrow B$ such that the order of the kernel is prime to $n$. If $A$ and $B$ are of the same genus, then $A \times A \cong B \times C$, where $C$ is another torus of the same genus. Two is in the stable range for the endomorphism ring of a torus, so if $A, B$, and $C$ 
are tori, and $A \times A \times B \cong A \times C$, then $C \cong A^{\prime} \times B$, where $A^{\prime} \times A \cong$ $A \times A$. If $\operatorname{End}(A)$ is commutative, and, in particular, if $A$ has (complex) dimension one, then $C \cong A \times B$. If $A, B$, and $C$ are tori and $A \times C \cong A \times B$, then $B$ and $C$ are of the same genus, and, finally, $B$ and $C$ are of the same genus if and only if for some positive integer $n, B^{n} \cong C^{n}$.

Most of the above theorem follows immediately from the preceding remarks. We regard the stated interpretation of the notion of genus as obvious. The fact that if a torus has complex dimension one then its endomorphism ring is commutative follows from the fact that it is a subring of the ring of complex numbers, and the result on cancelling tori with commutative endomorphism ring follows from Vasconcelos's result $[33,1.5]$ that if $R$ is a commutative ring and $R \oplus R \cong R \oplus I$ then $R \cong I$.

\section{REFERENCES}

1. D. Arnold, Genera and decompositions of torsion free modules, in "Abelian Group Theory" (Proceedings of the 2nd New Mexico State University Conference, 1976), Springer Lecture Notes in Math. 616, Berlin, 1977, 197-218.

2. D. Arnold and E. L. Lady, Endomorphism rings and direct sums of torsion free Abelian groups, Trans. Amer. Math. Soc., 211 (1975), 225-237.

3. H. Bass, K-Theory and Stable Algebra, Pub. Math. I.H.E.S., 22 (1964), 5-60.

4. P. M. Cohn, The complement of a finitely generated direct summand of an Abelian group, Proc. Amer. Math. Soc., 7 (1956), 520-521.

5. P. Crawley, The cancellation of torsion Abelian groups in direct sums, J. Algebra, 2 (1965), 432-442.

6. D. Eisenbud and J. C. Robson, Modules over Dedekind prime rings, J. Algebra, 16 (1970), 67-85.

7. D. Estes and J. Ohm, Stable range in commutative rings, J. Algebra, 7 (1967), 343-362.

8. E. G. Evans, Jr., Krull-Schmidt and cancellation over local rings, Pacific J. Math., 46 (1973), 115-121.

9. L. Fuchs, On a substitution property of modules, Monatshefte f. Math., 75 (1971), 198-204.

10. L. Fuchs and F. Loonstra, On the cancellation of modules in direct sums over Dedekind domains, Indag. Math., 33 (1971), 163-169.

11. K. Goodearl, Ring Theory; Nonsingular Rings and Modules, Marcel Dekker, New York, 1976.

12. - Power cancellation of group and modules, Pacific J. Math., 64 (1976), 387-411.

13. K. Goodearl and R. B. Warfield, Jr., Algebras over zero-dimensional rings, Math. Ann., 223 (1976), 157-168.

14. R. Gordon and J. C. Robson, Krull dimension, Mem. Amer. Math. Soc., 133 (1973).

15. R. Heitman, Generating ideals in Prufer domains, Pacific J. Math., 62 (1976), 117126.

16. M. Henriksen, On a class of regular rings which are elementary divisor ring, Archiv der Math., 24 (1973), 133-141.

17. H. Jacobinski, Genera and decompositions of lattices over orders, Acta Math., 121 (1968), 1-29. 
18. A. V. Jategaonkar, Left Principal Ideal Rings, Springer Lecture Notes in Math. 123, Berlin, 1970.

19. V. Jategaonkar, Multiplicative analogue of Weyl algebras, Notices Amer. Math. Soc., 23 (1976), A-566.

20. B. Jónsson, On direct decompositions of torsion-free Abelian groups, Math. Scand., 7 (1959), 361-371.

21. I. Kaplansky, Projective Modules, Ann. Math., 68 (1958), 372-377.

22. - Commutative Rings, Allyn and Bacon, Boston, 1970.

23. E. L. Lady, Summands of finite rank torsion-free Abelian groups, J. Algebra, 32 (1974), 51-52.

24. - Nearly isomorphic torson-free Abelian groups, J. Algebra, 35 (1975), 235-238.

25. G. S. Monk, A characterization of exchange rings, Proc. Amer. Math. Soc., 35 (1972), 344-353.

26. B. Muller, Localization of noncommutative Noetherian rings at semiprime ideals, McMaster University Lecture Notes, 1974.

27. J. C. Robson, Noncommutative Dedekind rings, J. Algebra, 9 (1968), 249-265.

28. J. T. Stafford, Stable structure of noncommutative Noetherian rings, J. Algebra, 47 (1977), 244-267.

29. R. Swan, Projective modules over group rings and maximal orders, Ann. Math., (2) 76 (1962), 55-61.

30. - The number of generators of a module, Math. Z., 102 (1967), 318-322.

31. - Algebraic K-Theory, Springer Lecture Notes in Math. 76, Berlin, 1968.

32. H. P. F. Swinnerton-Dyer, Analytic Theory of Abelian Varieties, Cambridge, Cambridge University Press, 1974.

33. W. V. Vasconcelos, On local and stable cancellation, An. Acad. Brasil. Ci., 37 (1965), 389-393.

34. L. M. Vasershtein, Stable rank of rings and dimensionality of topological spaces, J. Functional Anal. Appl., 5 (1971), 102-110.

35. E. A. Walker, Cancellation in direct sums of groups, Proc. Amer. Math. Soc., 7 (1956), 898-902.

36. - Quotient categories and quasi-isomorphisms of Abelian groups, Proc. Colloq. Abelian Groups, Tihany (Hungary) 1963 (Publ. House of the Hung. Acad. Sci., Budapest, 1964), 147-162.

37. R. B. Warfield, Jr., Exchange rings and decompositions of modules, Math. Ann., 199 (1972), 31-36.

38. - Genus and cancellation for groups with finite commutator subgroups, J. Pure and Appl. Algebra, 6 (1975), 125-132.

39. - Notes on cancellation, stable range, and related topics, University of Washington, 1975.

40. D. B. Webber, Ideals and modules of simple Noetherian hereditary rings, J. Algebra, 16 (1970), 239-242.

41. H. Zassenhaus, Orders as endomorphism rings of modules of the same rank, J. London Math. Soc., 42 (1967), 180-182.

Received July 27, 1977.

The UNIVERSity OF WASHington

Seattle, WA 98195 



\section{PACIFIC JOURNAL OF MATHEMATICS}

\section{EDITORS}

DONALD BABBITT (Managing Editor)

University of California

Los Angeles, CA 90024

Hugo RossI

University of Utah

Salt Lake City, UT 84112

C. C. MOORE and ANDREW OGG

University of California

Berkeley, CA 94720

\section{J. DugundjI}

Department of Mathematics

University of Southern California

Los Angeles, CA 90007

R. FinN and J. Milgram

Stanford University

Stanford, CA 94305

\section{ASSOCIATE EDITORS}
E. F. BeCKENBACH
B. H. Neumann
F. WoLF
K. YoSHIDA

\section{SUPPORTING INSTITUTIONS}

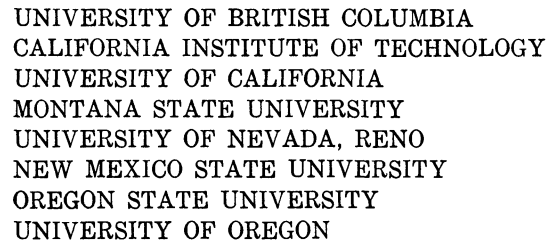

UNIVERSITY OF BRITISH COLUMBIA CALIFORNIA INSTITUTE OF TECHNOLOGY UNIVERSITY OF CALIFORNIA MONTANA STATE UNIVERSITY UNIVERSITY OF NEVADA, RENO NEW MEXICO STATE UNIVERSITY OREGON STATE UNIVERSITY UNIVERSITY OF OREGON

\author{
UNIVERSITY OF SOUTHERN CALIFORNIA \\ STANFORD UNIVERSITY \\ UNIVERSITY OF HAWAII \\ UNIVERSITY OF TOKYO \\ UNIVERSITY OF UTAH \\ WASHINGTON STATE UNIVERSITY \\ UNIVERSITY OF WASHINGTON
}

The Supporting Institutions listed above contribute to the cost of publication of this Journal, but they are not owners or publishers and have no responsibility for its content or policies.

Mathematical papers intended for publication in the Pacific Journal of Mathematics should be in typed form or offset-reproduced, (not dittoed), double spaced with large margins. Please do not use built up fractions in the text of the manuscript. However, you may use them in the displayed equations. Underline Greek letters in red, German in green, and script in blue. The first paragraph or two must be capable of being used separately as a synopsis of the entire paper. Please propose a heading for the odd numbered pages of less than 35 characters. Manuscripts, in triplicate, may be sent to any one of the editors. Please classify according to the scheme of Math. Reviews, Index to Vol. 39. Supply name and address of author to whom proofs should be sent. All other communications should be addressed to the managing editor, or Elaine Barth, University of California, Los Angeles, California, 90024.

50 reprints to each author are provided free for each article, only if page charges have been substantially paid. Additional copies may be obtained at cost in multiples of 50 .

The Pacific Journal of Mathematics is issued monthly as of January 1966. Regular subscription rate: $\$ 84.00$ a year (6 Vols., 12 issues). Special rate: $\$ 42.00$ a year to individual members of supporting institutions.

Subscriptions, orders for numbers issued in the last three calendar years, and changes of address shoud be sent to Pacific Journal of Mathematics, P.O. Box 969, Carmel Valley, CA 93924, U.S.A Old back numbers obtainable from Kraus Periodicals Co., Route 100, Millwood, NY 10546.

PUBLISHED BY PACIFIC JOURNAL OF MATHEMATICS, A NON-PROFIT CORPORATION

Printed at Kokusai Bunken Insatsusha (International Academic Printing Co., Ltd.). 8-8, 3-chome, Takadanobaba, Shinjuku-ku, Tokyo 160, Japan. 


\section{Pacific Journal of Mathematics}

\section{Vol. 91, No. 2 December, 1980}

Victor P. Camillo and Julius Martin Zelmanowitz, Dimension modules ... . . 249

Yonina S. Cooper, Stable sequences in pre-abelian categories ........... 263

Chandrakant Mahadeorao Deo and H. Ship-Fah Wong, On Berry-Esseen approximation and a functional LIL for a class of dependent random fields.........................................

H. P. Dikshit and S. N. Dubey, $|C, 1|$ summability of series associated with

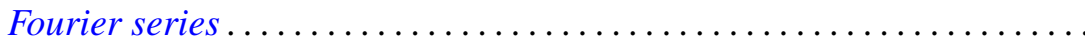

M. Edelstein, On the homomorphic and isomorphic embeddings of a semiflow into a radial flow.

Gilles Godefroy, Compacts de Rosenthal ..................... 293

James Guyker, Commuting hyponormal operators ................ 307

Thomas Eric Hall and Peter R. Jones, On the lattice of varieties of bands of

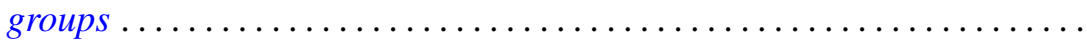

Taqdir Husain and Saleem H. Watson, Topological algebras with orthogonal Schauder bases ....................................

V. K. Jain, Some expansions involving basic hypergeometric functions of two variables. . .

Joe W. Jenkins, On group actions with nonzero fixed points ........... 363

Michael Ellsworth Mays, Groups of square-free order are scarce ........ 373

Michael John McAsey, Canonical models for invariant subspaces... 377

Peter A. McCoy, Singularities of solutions to linear second order elliptic partial differential equations with analytic coefficients by approximation methods...

Terrence Millar, Homogeneous models and decidability.

Stephen Carl Milne, A multiple series transformation of the very well poised

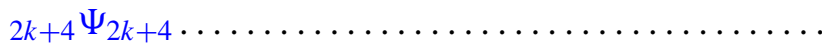

Robert Olin and James E. Thomson, Irreducible operators whose spectra are spectral sets...

Robert John Piacenza, Cohomology of diagrams and equivariant singular

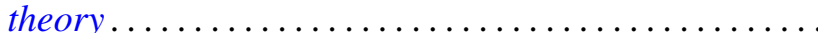

Louis Jackson Ratliff, Jr., Integrally closed ideals and asymptotic prime divisors

Robert Breckenridge Warfield, Jr., Cancellation of modules and groups and stable range of endomorphism rings.................

B. J. Day, Correction to: "Locale geometry" ...............

Stanley Stephen Page, Correction to: "Regular FPF rings" ... 